\title{
Successful hospital readmission reduction initiatives: Top five strategies to consider implementing today
}

\author{
Bita A. Kash ${ }^{1,2}$, Juha Baek ${ }^{* 1,2}$, Ohbet Cheon ${ }^{2}$, Nana E. Coleman ${ }^{3}$, Stephen L. Jones ${ }^{2}$ \\ ${ }^{1}$ School of Public Health, Texas A\&M University, College Station, Texas, United States \\ ${ }^{2}$ Center for Outcomes Research, Houston Methodist Research Institute, Houston Methodist Hospital, Houston, Texas, United \\ States \\ ${ }^{3}$ Department of Pediatrics, Baylor College of Medicine, Houston, Texas, United States
}

Received: August 9, 2018

DOI: $10.5430 /$ jha.v7n6p16
Accepted: October 4, 2018

Online Published: October 22, 2018

\begin{abstract}
Only one quarter of U.S. hospitals demonstrated low enough levels of 30-day readmission rates to avoid penalties imposed by the Hospital Readmissions Reduction Program (HRRP) in 2016. Previous work describes interventions for reducing hospital readmission rates; however, without a comprehensive analysis of these interventions, healthcare leaders cannot prioritize strategies for implementation within their healthcare environment. This comparative study identifies the most effective interventions to reduce unplanned 30-day readmissions. The MEDLINE-PubMed database was used to conduct a systematic review of existing literature about interventions for 30-day readmission reduction published from 2006 through 2017. Data were extracted on hospital type, setting, disease type, intervention type, study sample, and impact level. Of 4,886 citations, 508 articles were reviewed in full-text, and 90 articles met the inclusion criteria. Based on the three analytic methodologies of means, weighted means, and pooled estimated impact level, the most effective interventions to reduce unplanned 30-day admissions were identified as collaboration with clinical teams and/or community providers, post-discharge home visits, telephone follow-up calls, patient/family education, and discharge planning. Commonly, all five interventions identify patient level engagement for success. The findings reveal the need for shared accountability towards desired outcomes among health systems, providers, and patients while providing hospital leaders with actionable strategies that can effectively reduce 30-day readmission rates.
\end{abstract}

Key Words: 30-day readmission rate, Hospital readmission reduction initiative, Systematic review, Values-based medicine

\section{INTRODUCTION}

A significant number of hospitals in the U.S. will continue to be subject to penalties, as imposed by the Hospital Readmissions Reduction Program (HRRP) in 2016, due to unplanned 30-day readmissions. According to the latest report of the Centers for Medicare and Medicaid Services (CMS), only about one quarter (799 out of over 3,400) of all hospitals subject to the HRRP have demonstrated low/acceptable readmission rates to avoid any penalties. ${ }^{[1]}$ The HRRP was cre- ated under the Affordable Care Act, which despite present scrutiny and potential redesign, remains an important determinant of hospital reimbursements. Accordingly, targeted goals and interventions for the prevention of avoidable hospital readmissions will remain an important metric to avoid penalties on hospital reimbursement.

Earlier work has suggested that effective readmission reduction strategies ensure sufficient patient and caregiver resources — individual, social, and environmental—are avail-

\footnotetext{
${ }^{*}$ Correspondence: Juha Baek; Email: jbaek@sph.tamhsc.edu; Address: School of Public Health, Texas A\&M University, College Station, Texas,
} United States. 
able at discharge to minimize undue "burden" on the patient, which would result in poor outcomes, including unplanned readmission. ${ }^{[2]}$ Similarly, current literature examining strategies aimed at the reduction of hospital-acquired conditions as a comparable metric, affirms that longitudinal success in the improvement of health outcomes is both a multi-faceted and patient-centered process. ${ }^{[3]}$

A mitigating approach to avoidable readmissions additionally requires organizational engagement and capacity for change implementation. ${ }^{[4,5]}$ As an example, the importance of system-level transitional care processes in helping patients to sustain good health beyond the acute care setting has recently been highlighted. ${ }^{[6]}$ Interventions designed to prevent hospital readmissions among various patient populations that transition from an acute care setting to a post-acute care setting are often called transitional care interventions. ${ }^{[6]}$ Such strategies have been the subject of four noteworthy papers published in the last six years, which denote the impact of transitional care interventions on 30-day hospital readmissions. ${ }^{[2,4-6]}$

While these studies describe various interventions for reducing hospital readmission rates, they do not provide a comprehensive nor comparative analysis of the common characteristics of those interventions which are most effective, to enable health system leaders to prioritize which strategies to implement within their healthcare environment. Today's hospital leaders must consider: how do we prioritize finite resources to achieve the largest possible impact on readmission rates? Strategic prioritization of interventions to reduce unplanned 30-day readmissions suggests that patient-driven methods, grounded in the framework of values-oriented medicine, are most effective. This paper focuses on providing hospital leaders with a summary of evidence-informed successful strategies for readmission reduction, using three analytic methodologies.

\section{Methods}

\subsection{Data sources and study selection}

The database of MEDLINE-PubMed was used for searching English language peer-reviewed articles published from January 2006 to January 2017 to give an overview of trends in readmission interventions over the last decade, acknowledging the fact that readmission penalties were implemented in 2010. The first-pass filter utilized the following search terms related to readmission reduction interventions: "hospital readmission", "readmission rate", "re-hospitalization", "30-day readmission", "readmission prevention", "unplanned readmission", "avoidable readmission", "unnecessary readmission", "readmission strategy", "readmission program", "readmission intervention", "readmission initiative", and

Published by Sciedu Press "readmission treatment". Since care coordination and transitions of care are closely related to readmission reduction strategies, additional search terms were selected pertaining to care coordination or transitions of care such as "care coordination", "cost reduction", "coordinating care", "transitions of care", "care transition", "transitional care", "pre-discharge care", and "post-discharge care". Each search term was combined with the term of "readmission rate" to limit the scope of search only related to readmission. The search was conducted according to the guidelines of Preferred Reporting Items for Systematic Review and Meta Analysis Protocols. ${ }^{[7]}$

The review process was conducted in three phases by two reviewers as outlined in Figure 1. In the first phase, the researchers independently evaluated titles and abstracts of citations, excluding qualitative or case studies, review articles, non-peer reviewed journal articles, and studies irrelevant to a readmission reduction program. The second phase included full-text screening based on the following inclusion criteria: 1) including intervention(s) to reduce readmission rates; 2) using quantitative analysis with statistical methods; 3) having statistically significant results of readmission reduction; and 4) readmission rate as a primary outcome. Disagreements about inclusion or exclusion was reconciled by group discussion among the authors. In the third phase, the researchers selected articles that were related to only 30day readmissions, since some articles selected in the second phase included 60-day and 90-day readmissions.

\subsection{Data abstraction and measurement}

From the final list of included articles, the following data elements were extracted for analysis: hospital type, disease type, timing of intervention, number of key activities, type of intervention, name and description of program, setting, and impact level in readmission rate. The hospital type included five categories: academic health center, integrated system, for-profit, nonprofit, or others. Based on diseases of populations in the reviewed studies, the disease type was categorized by eight groups: heart-related, lung-related, mental, joint-related, and blood-related diseases, obesity/diabetes, others, and no specific diseases. Any study that reported a readmission reduction intervention using more than two disease categories was coded using multiple disease categories. Therefore, disease categories were not treated as mutually exclusive in this paper. The type of intervention was categorized using 12 groups following commonly reported categorization methodologies..$^{[2,8-10]}$ A description of these 12 intervention categories is presented in Table 1. The hospital settings included inpatient, outpatient, and community/home. The timing of intervention was categorized by pre-discharge, post-discharge, and bridging the transition. ${ }^{[10]}$ 
Lastly, the differences in readmission rates between an intervention group and a control group were recorded to calculate the impact level of each intervention.

\subsection{Data analysis and quality assessment}

To identify the most effective readmission reduction initiatives, three methodologies were used to analyze the impact of 12 interventions on 30-day readmission rates: 1) mean, 2 ) weighted mean, and 3) pooled estimated impact level. First, the average percentage of differences in 30-day readmission rates was calculated between before and after implementing each intervention in the studies. This term is referred as 'impact level' since the average percentage of differences indicates the population-level effects of each intervention on 30-day readmissions, which allows researchers to identify the most effective interventions on readmission rates on average. However, any given intervention's impact on readmission rates can vary based on the sample size. Since the selected studies have a wide range of the number of participants across the types of interventions, the readmission rates were weighted by sample size based on the premise that larger sample sizes tend to provide greater degrees of confidence for estimations when other things are equal. Following the methods of Bradburn, Deeks, and Altman, ${ }^{[11]}$ the weight values were calculated based on each study's sample size proportions among overall selected studies' samples and then multiplied it with the original mean values to calculate the weighted mean of readmission rates. ${ }^{[12,13]}$

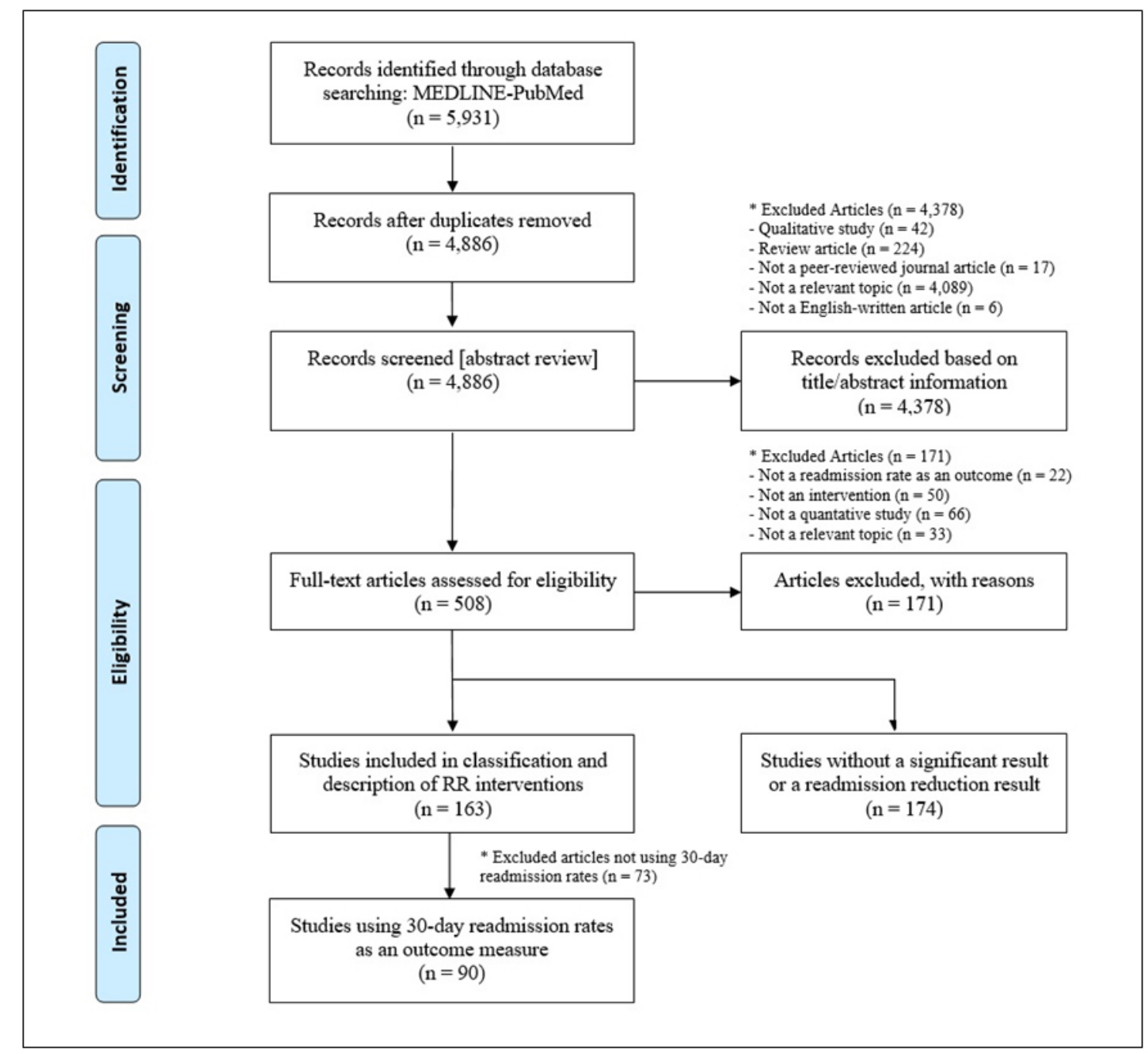

Figure 1. Flow diagram of review process

A meta-analysis was performed using Mantel-Haenszel fixedeffect model (metan in StataCorp. 2015. Stata Statistical Software: Release 14. College Station, TX: StataCorp LP) to calculate the pooled estimated impact level, which indicates the estimated impact of each intervention on readmission rates considering variance of the overall odd ratios. This method provides an alternative weighting scheme for analyzing the differences in 30-day readmission rates before and 
after taking interventions. The Mantel-Haenszel model can be more robust when data are sparse by producing similar estimates to the inverse variance methods. ${ }^{[14]}$ Based on the results from each methodology, the most effective interventions were organized by rank order selecting the top six interventions. Then the top five overlapping interventions across all three methodologies were selected as most promising interventions. Thereafter, authors sought to identify common characteristics among these top five interventions in order to provide a more meaningful assessment of the "impact" of these strategies on patient outcomes. The study was formally exempted by our institution's Institutional Review Board since it does not meet the definition of human subject research.

Table 1. Description, number and total sample size of interventions

\begin{tabular}{|c|c|c|c|c|}
\hline & Intervention & Description & $\begin{array}{c}\text { Number } \\
(\%)\end{array}$ & $\begin{array}{c}\text { Total } \\
\text { Sample Size }\end{array}$ \\
\hline 1 & $\begin{array}{l}\text { Collaboration/coordination } \\
\text { with other clinical teams, } \\
\text { PCP or community-based } \\
\text { programs }\end{array}$ & $\begin{array}{l}\text { A variety of activities of a multidisciplinary team or group that includes diverse } \\
\text { professions, such as nurses, pharmacists, and physicians, for discharge; Working } \\
\text { together for effective discharge process for patients with other clinical teams } \\
\text { and/or community-based programs or services; Engagement with primary care } \\
\text { physicians in communication about patient status }\end{array}$ & $\begin{array}{c}57 \\
(18 \%)\end{array}$ & 298,356 \\
\hline 2 & Home visits & $\begin{array}{l}\text { Physical visits by healthcare provider(s) to patient's home; Regular home visits } \\
\text { by profession(s), such as nurse, pharmacist, physician, and a multidisciplinary } \\
\text { team, for educating a patient, strengthening self-care instructions, and/or } \\
\text { performing physician examination }\end{array}$ & $\begin{array}{c}17 \\
(5 \%)\end{array}$ & 64,441 \\
\hline 3 & Telephone follow-up & $\begin{array}{l}\text { Use of telephone or videophone for communication initiated after discharge; } \\
\text { Monitoring or self-care management by using telephone for post-discharge } \\
\text { follow-up; Providing advice and education to patients and managing their } \\
\text { symptoms or clinical status by telephone call }\end{array}$ & $\begin{array}{c}43 \\
(14 \%)\end{array}$ & 251,011 \\
\hline 4 & $\begin{array}{l}\text { Patient/family education } \\
\text { (coaching) }\end{array}$ & $\begin{array}{l}\text { Patient-directed education or coaching to diagnosis or treatment rationale to } \\
\text { increase patient/family's knowledge and enhance their involvement in care; } \\
\text { Patient or family education delivered before, after, or at discharge by a variety of } \\
\text { means, such as in-person, interactive CD-ROM, MP3, video, or telephone }\end{array}$ & $\begin{array}{c}48 \\
(15 \%)\end{array}$ & 100,673 \\
\hline 5 & $\begin{array}{l}\text { Medication intervention } \\
\text { (medication reconciliation) }\end{array}$ & $\begin{array}{l}\text { Medication reconciliation or special education conducted by pharmacist and/or } \\
\text { nurse, which is aimed at improving medication understanding or adherence } \\
\text { before discharge; A review of the patient's medications by a pharmacist with a } \\
\text { view of improving the patient's knowledge of, and compliance with, the } \\
\text { treatment regimen, identifying medication, drug reactions, or interactions }\end{array}$ & $\begin{array}{c}37 \\
(12 \%)\end{array}$ & 62,310 \\
\hline 6 & Discharge planning & $\begin{array}{l}\text { Formalizing an approach to prepare for discharge; Establishing a transitional } \\
\text { care plan before discharge; Ensuring communication between the medical team } \\
\text { members and/or evaluating the patient's support network and post-discharge } \\
\text { services; Evaluating patients' abilities of self-care and household environments }\end{array}$ & $\begin{array}{c}25 \\
(8 \%)\end{array}$ & 77,815 \\
\hline 7 & Follow-up appointments & $\begin{array}{l}\text { Scheduling a follow-up visit with patients during their hospital stay; Helping } \\
\text { patients and/or families (caregivers) to make follow-up appointments before } \\
\text { discharge }\end{array}$ & $\begin{array}{c}36 \\
(12 \%)\end{array}$ & 192,296 \\
\hline 8 & $\begin{array}{l}\text { Telemonitoring } \\
\text { (Telehomecare) }\end{array}$ & $\begin{array}{l}\text { Use of remote technology designed for patients to transmit objective measures of } \\
\text { health status with or without connected subjective assessment; Remote } \\
\text { monitoring of physiologic data with digital, broadband, satellite wireless, or } \\
\text { Bluetooth transmission to a monitoring center, with or without remote clinical } \\
\text { visits; Participation in health information exchange (HIE), especially use of } \\
\text { electronic documentation, to improve care coordination }\end{array}$ & $\begin{array}{c}21 \\
(7 \%)\end{array}$ & 78,635 \\
\hline 9 & $\begin{array}{l}\text { Guideline implementation } \\
\text { for clinicians }\end{array}$ & $\begin{array}{l}\text { Adoption of and adherence to guidelines including rules or regulations about } \\
\text { clinical treatment; Compliance with guidelines regarding staffing like work-hour } \\
\text { restrictions }\end{array}$ & $\begin{array}{c}4 \\
(1 \%)\end{array}$ & 18,310 \\
\hline 10 & Rehabilitation intervention & $\begin{array}{l}\text { Patient-directed rehabilitation efforts that are not entirely diagnosis specific but } \\
\text { aimed at improving functional status; Participating in a supervised exercise } \\
\text { training program at the hospital's gym or individual home exercise program }\end{array}$ & $\begin{array}{c}4 \\
(1 \%)\end{array}$ & 1,743 \\
\hline 11 & $\begin{array}{l}\text { Clinical intervention/ } \\
\text { medical device }\end{array}$ & $\begin{array}{l}\text { Implementation of certain drug or surgery treatment; Use of an innovative } \\
\text { medical device in a surgery or medical care to improve clinical outcomes }\end{array}$ & $\begin{array}{c}13 \\
(4 \%)\end{array}$ & 118,255 \\
\hline 12 & $\begin{array}{l}\text { In-hospital management } \\
\text { units }\end{array}$ & $\begin{array}{l}\text { Hospital wards, staffed by doctors, nurses and other health professionals for } \\
\text { diagnostic assessment, therapy, rehabilitation, and placement of patients to } \\
\text { intensify post discharge care, identify effective community services, and } \\
\text { enhance primary care access; Use of a unit to improve patient management in } \\
\text { hospital }\end{array}$ & $\begin{array}{c}8 \\
(3 \%)\end{array}$ & 37,772 \\
\hline
\end{tabular}

Note. Since some of studies include more than two intervention programs (as bundled programs), some of participants in each intervention can be overlapped across intervention categories. Thus, the sum of each column can be greater than the total number of participants $(608,517)$ in this review. The types of intervention and their descriptions are derived from Kash et al. ${ }^{[15]}$ with the permission. 


\section{RESUlts}

The initial search resulted 4,886 unduplicated articles (see Figure 1). After abstract and title screening, 508 articles $(10.3 \%)$ were selected for full-text review. In the second phase, 163 articles (32\%) were selected based on the inclusion criteria. After the third phase, 90 studies reporting only 30 -day readmission rates as an outcome $(55 \%)$ were selected. Major characteristics of successful readmission reduction programs were profiled by intervention. The most frequently reported interventions were collaboration (18\%), education (15\%), telephone follow-up (14\%), medication interventions (12\%), and follow-up appointment (12\%) (see Table 1). Table 2 shows the results of the comparison of impact on 30-day readmissions among 12 interventions. The range of impact level on 30-day readmissions varied in each intervention. The authors used results of the three methodologies described to identify the "top five" interventions.

Table 2. Comparison of impact on 30-day readmission rates among 12 interventions using three methodologies

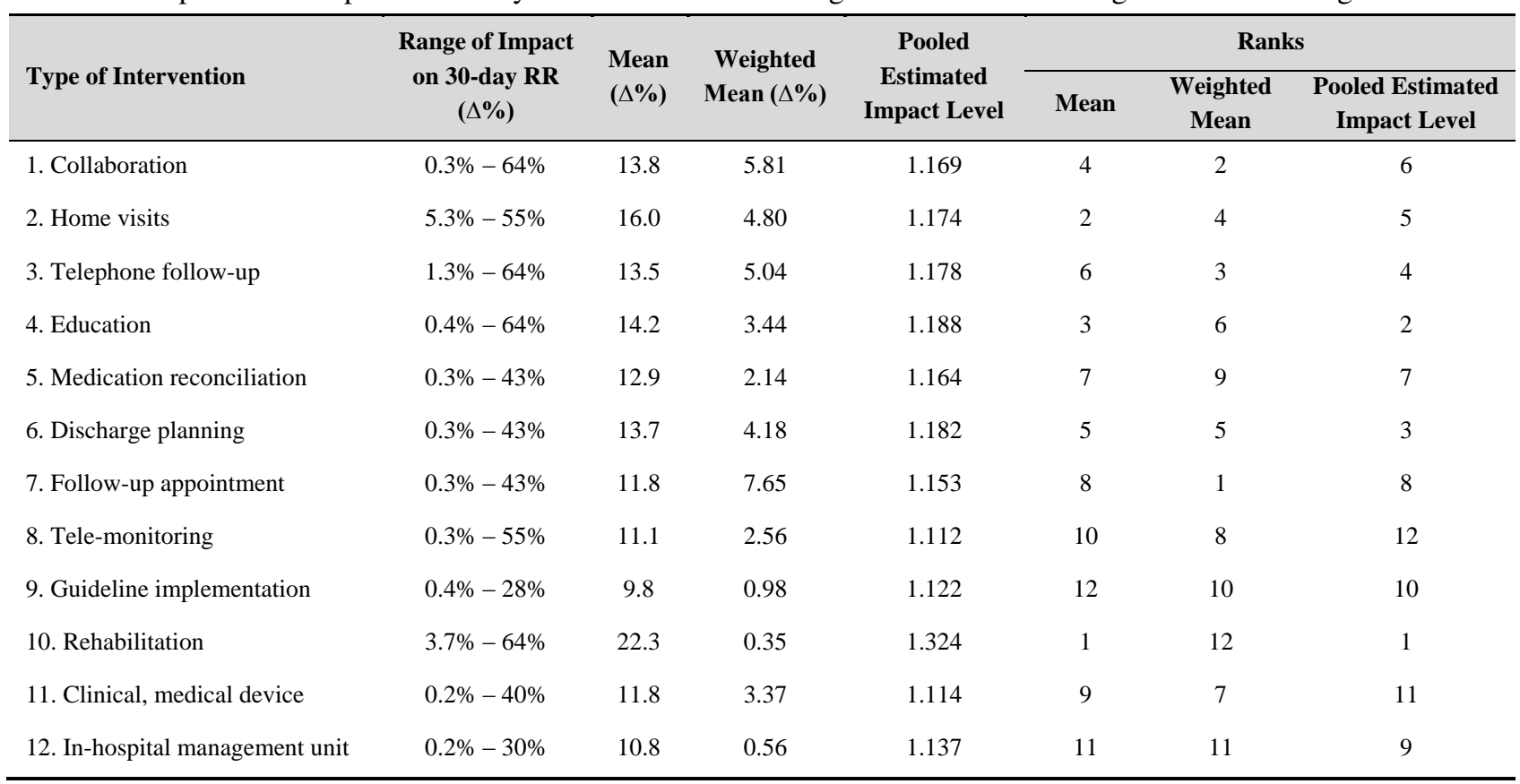

Note. All pooled estimated impact levels are statistically significant at $1 \%$ confidence level.

According to the first methodology, mean values, the intervention with the highest mean reduction in 30-day readmission rates was rehabilitation (22.3) and the lowest mean was guideline implementation (9.8). The top six interventions based on unweighted means were rehabilitation, home visits, education, collaboration, discharge planning, and telephone follow-up.

The second methodology, weighted means, produced slightly different results: follow-up appointment had the highest value (7.65), whereas rehabilitation had the lowest value (0.35). The top six interventions by weighted mean included follow-up appointment, collaboration, telephone follow-up, home visits, discharge planning, and education. The rank order of classified interventions is strongly influenced by the sample size of each study and the total number of participants who were subject to each type of interventions across all reviewed studies.

Based on the third methodology, the pooled estimated impact level, rehabilitation had the highest level of impact on readmission rates (1.324) while tele-monitoring had the lowest impact (1.112). The pooled estimated impact level resulted in the following rank order: 1) rehabilitation, 2) education, 3) discharge planning, 4) telephone follow-up, 5) home visits, and 6) collaboration. The findings also indicate that all Mantel-Haenszel pooled estimated impact levels are statistically significant at the $1 \%$ significant level $(p$-value $<.001)$.

Considering all three statistical methodologies in aggregate, the research team found that five interventions - collaboration, home visits, telephone follow-up, education, and discharge planning - consistently demonstrated high impact on the reduction of 30-day readmissions. Furthermore, when evaluating the three statistical methods of assessing the impact of various readmission prevention strategies independently, these five interventions were included among the top six interventions. It was consistently noted that tele-monitoring, guideline implementation, medical device, and in-hospital 
management units demonstrated the lowest impact level on readmission rates.

This study stratified the impact of the described avoidable 30-day readmission prevention strategies as "low, medium, and high impacts", to enable a more meaningful comparison of the effectiveness of each initiative. Each strategy was stratified using the observed percent change in readmission rates: low impact (0\%-9.99\%), medium impact (10\%-19.99\%) and high impact ( $20 \%$ or more). These cut-off points were used based on input from industry experts including chief medical officers, physician scientists and other clinical leaders at three hospital systems in the U.S. Figure 2 visualizes the percentages of studies by impact level in each intervention.
The findings indicate that home visits, discharge planning, education, and telephone follow-up significantly reduced 30day readmission rates while also showing a relatively high and significant impact level. More than 50\% of these four interventions show impact levels at greater than $10 \%$ (greater than medium level). Based on this result, the researchers found that the most effective interventions identified using the three statistical methodologies had the highest impact on 30-day readmission rates. Readmission reduction strategies described as having collaboration as a significant characteristic of the protocol comprised a relatively low proportion (46\%) of the interventions that stratified to the medium and high impact level groups.

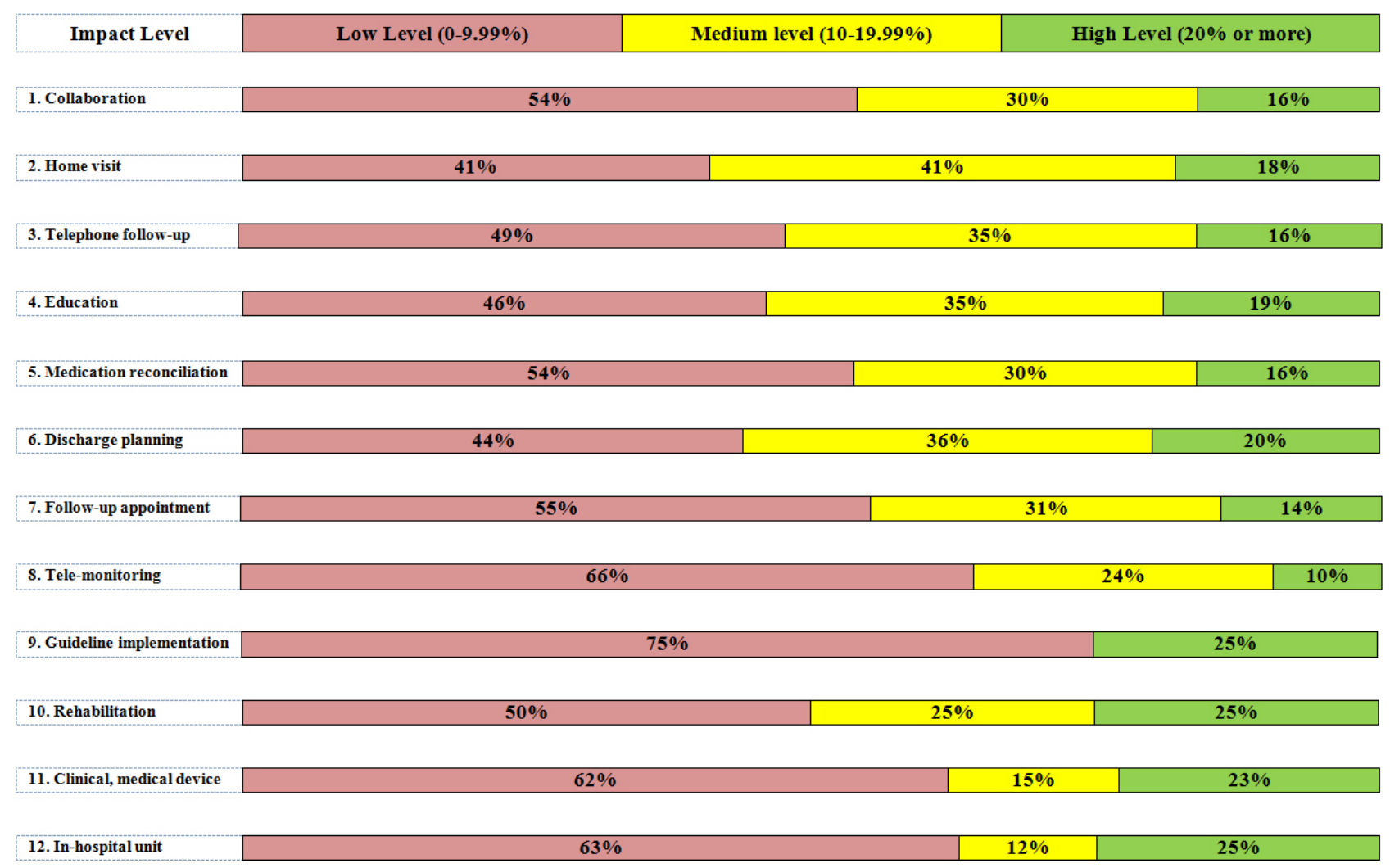

Figure 2. Impact levels of interventions

\section{Discussion}

\subsection{Discussion of the findings}

This study reports that collaboration, home visits, telephone follow-up, education, and discharge planning are the most frequently studied and the most successful strategies to reduce unplanned readmissions across varying statistical measures. While enhanced by organizational and system-level performance, the success of each initiative is primarily patientdriven. Shared accountability for clinical outcomes between patients and healthcare teams represents a modern conceptualization of healthcare practice, one in which patients, providers and health systems work together to optimize healthcare outcomes. ${ }^{[16,17]}$

Previous work has evaluated common characteristics among hospital readmission reduction strategies. Hansen et al. ${ }^{[10]}$ provide a general framework for categorizing various readmission reduction strategies, which informed this study's analysis. They report that effective pre-discharge interventions include patient education, medication reconciliation, and discharge planning. Post-discharge interventions include telephone follow-up, communication with ambulatory providers, and home visits. Bridging interventions include 
patient-centered discharge instructions. The results in this study confirm some of these findings.

Other authors have undertaken disease-specific analysis of the effectiveness of readmission reduction initiatives. In a 2013 meta-review article, Benbassat and Taragin ${ }^{[8]}$ consider the importance of community-based programs in reducing heart failure-related readmissions. A 2014 meta-analysis and systematic review by Feltner et al. ${ }^{[9]}$ describes effective hospital readmission reduction initiatives focused on congestive heart failure; specifically home-visit programs, multidisciplinary heart failure clinic interventions and structured telephone calls highlight the importance of care continuity and patient integration in the healthcare team in mitigating unplanned readmissions.

As noted previously, the work of Leppin et al. ${ }^{[2]}$ supports a patient-centered approach to reducing unplanned readmissions. The results of this study support this model for health outcome improvement, given the most frequently studied and most impactful readmission reduction strategies the authors identified also necessitate patient accountability for success This "values-based" approach to implementing the most impactful interventions aligns health system and provider values and goals with patient values and capacity.

While the authors present the most effective strategies in preventing hospital readmissions, it is important to frame the connection between the shared responsibilities of patient and provider in achieving this goal of care. The framework of values-based care provides such a heuristic, and was notably defined by Bill Fulford's formative work in this area as a "skills-based approach to consistently and intentionally incorporate patients' values into clinical decision making alongside best research evidence; it is a process to guide balanced decision-making where complex and/or conflicting values are involved" (p. 341-342). ${ }^{[18]}$ The research team would argue that in such a model of care, patients are actively empowered in their healthcare, consistent with the findings that patient-centered strategies to prevent readmission, are ultimately most successful.

\subsection{Limitations}

Most studies included in this review paper were conducted in single health systems spanning the spectrum of not-for-profit, academic, and for-profit systems and therefore raises the question of applicability across broader, more diverse and smaller, environments. Also, the three methodologies used in this study were developed by this research team and do not follow a prescribed methodological approach, as no validated scale exists to assess the impact level on readmissions. In addition, this study is not informed by arrangements for implementing readmission reduction interventions, or the costs incurred to implement them, given the restricted information in the selected studies. Future studies should consider identifying common protocols for implementing the top five interventions presented in this study. Furthermore, future studies could include cost-benefit analyses once information on implementation cost becomes available. Finally, this paper only presents results of successful readmission reduction interventions that have been reported in peer-reviewed literature between 2006 and 2017. Unlike other systematic reviews with a single intervention, this study compares the effectiveness of 12 types of interventions with various patient populations, settings, and diagnoses. Due to the relatively large heterogeneity and complexity of this study, the authors focused on studies reporting positive significant results only. A follow-up study will compare studies reporting negligible (statistically insignificant), no impact, or a negative impact on unplanned 30-day readmission rates as a means of validating the findings presented in this manuscript.

\subsection{Implications}

This study acknowledges the importance of both organizational and patient engagement in reducing the incidence of unplanned readmissions as a core performance measure for many hospitals. Despite reasonable evidence to support a distinct group of patient-centered strategies as most efficacious, further analysis of hospital readmissions data suggests that the best way to prevent hospital readmissions is to prevent the index admission in the first place, whenever possible. Inherently, emphasis on patient capacity as fostered through health literacy, preventive care, and collaborative partnership with the health system is equally important. Patients have a very substantial stake in readmission reduction strategies and accordingly should also be held accountable for hospital readmission outcome. What bears further study is the incentive to patients at an individual level for avoiding readmission, particularly when a patient's individual values do not align with those of the provider or health system.

\section{Conclusions}

The top five strategies with the highest impact on reducing readmission rates are all collaborative in nature and would likely benefit from a values-based medical practice model. Operationalizing values-based medical practice requires a multi-faceted and systematic approach to healthcare delivery redesign. This paradigm of practice fundamentally serves to enhance awareness of and to promote individual reflection on values while maintaining care delivery standards consistent with the best scientific evidence. Clinical teams must ensure that patients are cognizant of the risks and benefits associated with various treatment choices as their values and preferences 
are incorporated into the care plan. Implicit within this is a clear delineation of the roles and responsibilities of both patients and care providers alike within the healthcare team. In this model of care delivery, patients balance the "burden" of their health outcomes with the healthcare system. It is through this intricate partnership with patients that the top five interventions are best able to flourish.

\section{CONFlicts OF INTEREST Disclosure}

The authors declare they have no conflicts of interest.

\section{REFERENCES}

[1] Dickson V. Hospitals push readmissions fix in lame-duck Congress [Modern Healthcare website]. November 14, 2016. Available from: http://www.modernhealthcare.com/article/20161 114/NEWS/161119966

[2] Leppin AL, Gionfriddo MR, Kessler M, et al. Preventing 30-day hospital readmissions: a systematic review and meta-analysis of randomized trials. JAMA Internal Medicine. 2014; 174(7): 1095-1107. PMid: 24820131. https://doi.org/10.1001/jamainternmed. 2014.1608

[3] King HB, Kesling K, Birk C, et al. Leveraging the Partnership for Patients' Initiative to Improve Patient Safety and Quality Within the Military Health System. Military Medicine. 2017; 182(3): e1612e1619. PMid: 28290933. https://doi .org/10.7205/MILMED-D -16-00077

[4] Kash BA, Spaulding A, Gamm LD, et al. Leadership, culture, and organizational technologies as absorptive capacity for innovation and transformation in the healthcare sector. Change Management. 2014; 13(1): 1-13.

[5] Spaulding A, Kash BA, Johnson CE, et al. Organizational capacity for change in health care: Development and validation of a scale. Health Care Management Review. 2017; 42(2): 151-161. PMid:26587997. https://doi.org/10.1097/HMR.0000000000000096

[6] Naylor MD, Aiken LH, Kurtzman ET, et al. The importance of transitional care in achieving health reform. Health Affairs. 2011; 30(4): 746-754. PMid: 21471497. https://doi.org/10.1377/hlthaf f. 2011.0041

[7] Moher D, Shamseer L, Clarke M, et al. Preferred reporting items for systematic review and meta-analysis protocols (PRISMA-P) 2015 statement. Systematic Reviews. 2015; 4: 1. PMid: 25554246. https://doi.org/10.1186/2046-4053-4-1

[8] Benbassat J, Taragin MI. The effect of clinical interventions on hospital readmissions: a meta-review of published meta-analyses. Israel Journal of Health Policy Research. 2013; 2(1): 1. PMid: 23343012. https://doi.org/10.1186/2045-4015-2-1

[9] Feltner C, Jones CD, Cené CW, et al. Transitional care interventions to prevent readmissions for people with heart failure: A systematic re- view and meta-analysis. Annals of Internal Medicine. 2014; 160(11): 774-84. PMid: 24862840. https://doi.org/10.7326/M14-008 3

[10] Hansen LO, Young RS, Hinami K, et al. Interventions to reduce 30-day rehospitalization: a systematic review. Annals of Internal Medicine. 2011; 155(8): 520-528. PMid: 22007045. https: //doi.org/10.7326/0003-4819-155-8-201110180-00008

[11] Bradburn MJ, Deeks JJ, Altman DG. Metan-an alternative metaanalysis command. Stata Technical Bulletin. 1999; 8(44).

[12] Higgins JP, Thompson SG, Tierney J, et al. Presenting random effects meta-analyses: where are we going wrong. Paper presented at: International Cochrane Colloquium. 2001.

[13] Higgin J, Thompson S, Deeks J, et al. Measuring inconsistency in meta-analysis. British Medical Journal. 2003; 327: 557-560. PMid: 12958120. https://doi.org/10.1136/bmj .327.7414.557

[14] Robins J, Breslow N, Greenland S. Estimators of the MantelHaenszel variance consistent in both sparse data and large-strata limiting models. Biometrics. 1986; 311-323. PMid: 3741973. https : //doi.org/10.2307/2531052

[15] Kash BA, Baek J, Davis E, et al. Review of successful hospital readmission reduction strategies and the role of health information exchange. International Journal of Medical Informatics. 2017; 104: 97-104.

[16] Detsky AS. What patients really want from health care. JAMA. 2011; 306(22): 2500-2501. PMid: 22166610. https://doi.org/10.1 $001 /$ jama. 2011.1819

[17] Joffe S, Manocchia M, Weeks J, et al. What do patients value in their hospital care? An empirical perspective on autonomy centred bioethics. Journal of Medical Ethics. 2003; 29(2): 103-108. PMid: 12672891. https://doi.org/10.1136/jme.29.2.103

[18] Fulford K. Bringing together values-based and evidence-based medicine: UK Department of Health Initiatives in the 'Personalization' of Care. Journal of Evaluation in Clinical Practice. 2011; 17(2): 341-343. PMid: 21114716. http://dx . doi .org/10.1186 /1478-4505-9-20 